cooling is mixed with the iodoform in a mortar which has been sterilized by means of boiling water and formalin. The paraffin is added to the mixture at a temperature of $90^{\circ} \mathrm{C}$. and thoroughly mixed, then put into specially marle collapsible tubes with nozzle ends, holding 40 to 80 grams. The paraffin used should be semi-solid, odourless, and tasteless, melting point $45^{\circ}$ C., free from acidity, and carbonizable organic Impurities. The iodoform should be free from moisture, acids, and fixed impurities. It is also necessary that the bismuth be chemically pur that the temperature does not rise too high, otherwise nitrous umes will be evolved.

A paste made by this method is found to be fairly solid in consistency, shows no signs of separation or decomposition, and is perfectly sterile, as certified by Dr. Slade, bacteriologist to this infirmary.

Should a paste be required of a softer consistency the formula may be modified as follows?

\begin{tabular}{|c|c|c|c|c|}
\hline Iodoform & & & & 440 grams \\
\hline Bismuth subritrate & $\ldots$ & $\ddot{0}$ & $\ddot{\ldots}$ & 220 \\
\hline Paraftin base ... & M.S.A. & $\therefore$ & ... & 220 \\
\hline
\end{tabular}

Paraffin base :
Paraffin melting point 45
Paraffin liq., sp. gr. 880
$\cdots$
... 19 parts

.. 40 ,

Special tubes with keys, suitable for the above preparation, can be obtained from Whitelaw and Co., Saville Row, Newcastle on-Tyne, and tabes ready charged with Bipp sterilized according to my method are obtainable from the Numol Company, 3, College Street, Newcastle-on-Tynt.

Though this method has been used chiefly for war wounds it is applicable, as Case II shows, in civilian cases. My colleague, Dr. Samuel Whyllis, tells me that the method has revolutionized mastoid surgery, and I have a report from a London throat and ear surgeon telling me how much benefit had followed its use in his work-in mastoid cases.

\section{Summary of Technique.}

1. Under an anaesthetic, usually open ether, cover the wound with gauze wrung out of 1 to 20 carbolic acid, and clean the skin and the surrounding area with the same lotion.

2. Open the wound freely and, if possible, sufficiently to permit of inspection of its cavity. A guide-a finger is the best if the size of the wound permits of it, and if not a thick probe-should be introduced to the bottom of the wound and held there and fully exposed. In doing this special regard must be paid to nerve trunks and muscular branches of nerves, since the division of blood vessels, excepting the largest, and of muscles themselves does little harm as compared with that of the disability following nerve damage. Cleanse the cavity with dry sterile gauze mops, Volkmann's spoon, etc., and remove all foreign bodies.

3. Mop the surrounding skin and the wound cavity with methylated spirit and dry it.

4. Fill up the whole wound with Bipp, rub it well in with dry gauze. Then remove all excess, leaving only a thin covering over the wounded surface. Dress the wound with sterile gauze and cover all with an absorbent pad, which is held in position by sticking plaster and a bandage. This dressing requires no change for days or weeks if the patient is free from pain and constitutional disturbance. Should, liowever, discharge come through, the stained part must be soaked in spirit and a gauze dressing wrung out of the same applied as a further covering.

Redressing is very simply done. After removal of the old dressings the wound is covered with a dossil of wool soaked in spirit, and the sticky dirty-looking discharge is wiped off the surrounding skin until it is clean.

I am indebted to Colonel Adams for leave to publish the military cases.

REFERENOES.

${ }^{1}$ Lancet, August 12th, 1916; British Journal of Surgery, April, 1917. 2 Lancet, March 3rd, 1917.

Dr. Wardell Stiles of the United States Public Health Service urges careful examination of all recruits of the National Guard and National Army, especially from the south, for lfookworm. The parasite. was found in fortyseven among seventy-five recruits. Dr. Stiles calls attention to the great danger of carrying the infection from America to Europe.

\section{REPORT ON WOU.ND TREATMENT BY BRILLIANT GREEN PASTE.}

BY

Captain A. RENDLE SHORT, M.D., B.S., B.Sc., F.R.C.S., R.A.M.C.(T.F.),

SENIOR ASSISTANT SORGEON, BRISTOL ROYAL INFIRMARY;

Captain J. S. ARKLE, M.B., B.S., R.A.M.C.(T.C.), AND

Captain C. KING, M.B., B.C., R.A.M.C.(T.C.).

IT has been abundantly proved by experience that to secure efficient sterilization of an infected wound it is necessary to apply antiseptic remedies, not occasionally, but continuously. There are two means of obtaining this end. The one is the use of the Carrel method of frequent introduction of fluids through rubber tubes, and the other is the thorough application of a paste to every hole and corner of the wound, as exemplified by the popular bipp of Rutherford Morison. Both these methods have draw. backs. The Carrel technique needs much attention, and when the patient is travelling this may not always be secured. The soaked dressings need daily changing. The tubes are liable to come out and it may need an anaesthetic to replace them. In "strafe" times it may be frankly impossible to devote sufficient time to carry it out.

Bipp is much less trouble, but presents drawbacks of another sort. It is occasionally poisonous, either from the bismuth or the iodoform, or both. It may delay healing. when the stitches are taken out after secondary suture the wound may fly open. It leaves a residue in the tissues, opaque to the $x$ rays, which may give rise to trouble later. One of us has seen an unnecessary operation in England to remove iodoform from a hole in a tibia, suggesting in the skiagram a metallic fragment; and on another occasion, in civil practice, iodoform put into a tuberculous os calcis two year's before was mistadien for a sequestrum.

We have therefore been employing for some months the paste introduced by Captain Hey, after long research into many methods, consisting of brilliant green, boric acid, French challs, and liquid paraffin. It presents the following advantages:

1. It is non-poisonous. The symptoms of boric acid poisoning-persistent vomiting and a peculiar rashshould be watched for, but although in many cases we have used very large quantities of the paste we have seen no ill effect result from its use.

2. It is painless.

3. In the great majority of cases it almost completely sterilizes the wound in three days, so that secondary suture can be performed even in large wounds complicated by bony injury. Small, completely excised wounds can be primarily sutured.

4. The wound need only be dressed about once in four days.

5. No permanent residue is left to interfere with immediate healing.

Before applying the paste the wound must be thoroughly opened up and excised, and any foreign bodies removed. If this is not possible-for example, in a shocked patient with multiple wounds or with a long deep track exposing important structures-we prefer to use Carrel's method. The paste is only to be applied if it can be applied com. pletely. The wound ought to be dry and not bleeding, but a certain amount of oozing can be checked by a gauze plug (preferably paraffin gauze, which is easier to remove). The paste should be rubbed in thoroughly. It soon washes off the skin or gloves.

By this method we have been able with fair consistency to obtain healing within a fortnight of cases of compound fracture even when complicated by joint injury, big buttock wounds and deep muscle wounds. We have excised an elbow-joint full of pus, and obtained primary healing in a fortnight.

The usual procedure is to stain the track of the missile, excise and paste it, and either leave it open or insert a few central sutures. On the third or fourth day a smear of the deepest part of the wound is taken. If $B$. perfringens is absent, as is almost invariably the case, and the organisms are not more than six to the field, we completely close the wound by secondary suture. Often the 
wound shows no organisme at all. It is easier to count the organisms with this method than when bipp has been used. A little redness and swelling on the fourth day can usually be remedied completely by hot fomentations.

We find that Hey's method of staining the wound by injecting through a Carrel's tube with a 1 in 200 watery solution of brilliant green is a very valuable aid to complete excision.

In ten cises it will be observed that the method has failed of complete success. Of these, four (Cases 10, 17, 23, and 33) were hopeless from the beginning. One of them (Case 23) was an attempt to save a leg in which it was afterwards found that both tibial arteries were divided in addition to the presence of a big. muscular wound and a smashed tibia and fibula. The excision was inadequate owing to shock and consequent hurry, and it became necessary to amputate for gas gangrene. In another (Case 10), in which death followed within forty-eight hours from gas gangrene, the bad condition of the patient did not permit of a prolonged search for the foreign body. The appearance of the wound at the time of operation suggested early gas gangrene, which had already spread too far for complete excision. The other two (C)ases 17 and 33) are sufficiently described in the notes. In Case 43 the use of the paste resulted in clean healing wounds, death being due to other causes. In two other cases (8 and 9) proper excision of the wound was impossible and both suppurated. The other three cases of this group $(28,29,30)$ were large deep wounds, which did well for four or five days and then suppurated. Probably the original pasting is not effective after three days. These cases could have been kept clean by repasting under an anaesthetic.

Just now we are trying a flavine paste. Sufficient material is not yet available for a report. The results are so far very similar to those obtained with brilliant green.

The undermentioned series of cases is classified under three headings :

Class I : Remarkably successful, in that the wounds were very extensive and in general were com plicated by bone or joint injury.

Class II : Less serious cases giving perfectly satis. factory results.

Class III : Relatively unsuccessful.

The report inclúdes every case up to August 16th in which the paste was used. In all cases the wounds were stained, excised, and the foreign body removed on the day of admission. Any deviation from this procedure is recorded in the notes of cases.

SUMMARY OF CASES

Class I.-Remarkably Successful.

Case 1.-Pte. F. D.; admitted July 8th, 1917, with the following shell wounds: (1) Penetrating wound of left knee; (2) gutter wound of right leg, grazing patella; (3) multiple wounds of left thigh; (4) multiple wounds of arm, one penetrating elbow. Usual treatment, except thigh and arm, where wounds could only be partially excised.

July 13th. Secondary suture of wound of right leg.

July 20th. Elbow septic, other wounds clean. On July 25th the elbow was drained, and on August 2nd the elbow-joint formally excised for suppurative arthritis; paste was applied and the wound closed by primary suture with drainage. The drain was removed on Angust 6th and on the 12th the elbow reaction, the other wounds were firmly healed, and the patient was evacuated to the base.

Case 7.-Pte. H. T.; admitted July 11th, 1917, with shell wounds of leg (with compound comminuted fracture of tibia), thigh, and back.

July 18th. Leg wound repasted and sutured.

July 25th. Healing; slight serous discharge from tibia; no pus; other wounds satisfactory. Evacuated to base.

Case 15.-Pte. C.; admitted July 13th, 1917, with shell wound of knee. Operation twenty-four hours after injury; thigh swollen and inflamed; foreign body removed from popliteal surface of femur.

July 19th. Repasted and sutured, except small space in centre of wound.

July 25th. Healing; wound clean. Evacuated to base.

Case 16.-Pte. R. G.; admitted July 14th, 1917, with shell wound of buttock with divided gluteal artery. Repasted at intervals without anaesthetic.

July 25th. Skin edges freshened and sutured; glove drain. Drain removed two days later; wound clean.

July 30th. Healing. Evacuated to base. Report from base :

Dne stitch removed on arrival : rest of wound healed.
Case 31.-Pte. A. D.; admitted July 24th, 1917. Shell wound of forearm with compound fracture of radius. Primary suture. On the 30th the wound was healing, and the patient was evacuated to the base.

Case 32.-Pte. R. C.; admitted July 24th, 1917. Shell wound of leg with compound fracture of tibia. Repasted and sutured on the 27th. Three days later the wound was healing, and the patient was evacuated to the base.

August 3rd. Report from base : Wound healed.

Case 34.-Pte. B. C. ; admitted July 27th, 1917. Trench mortar wounds of neck, exposing vertebrae, and ankle, with compound fracture of tibia and division of posterior tibial vessels and nerve.

July 31 st. Secondary suture of neck; ankle wound repasted. Loss of skin too extensive for suture. Patient evacuated to the base a few days later with both wounds clean.

Case 35.-Pte. J. M. ; admitted July 28th, 1917. Shell wound of arm with compound fracture of humerus and divided brachial arters. Secondary suture was performed on August $2 \mathrm{nd}$; the stitches were removed on the loth, when the wound was healed. Two days later he was evacuated to the base.

Case 39.-Pte. W. C.; admitted July 29th, 1917. Bomb wound of knee (penetrating) with compound fracture of patella and superficial wound of chest wall. Patella sewn with catgut; partial primary suture.

August 11th. Both wounds healed; no rise of temperature; joint never swollen. Evacuated to base.

Case 40.-Pte. A. B. ; admitted July 30th, 1917. Shell wound of arm with compound comminuted fracture of radius and ulna. On August 3rd the outer wound was completely sutured; the inner wound as far as skin available.

August 12th. Outer wound healed and dry; inner wound clean, no pus. Evacuated to base.

Case 47.-Pte. R. W.; admitted July 3lst, 1917, with bomb wounds of buttock and thigh; buttock wound deep, 7 in. track. Secondary suture was performed on August $3 \mathrm{rd}$. On the 12th the wounds were healed, there was no suppuration, and the patient was evacuated to the base.

Case 48.-Lce.-Cpl. A. C. ; adnitted July 31st, 1917, with bomb wound of thigh; track 17 in. long through muscles; patient very collapsed on admission.

August 4th. Lower half of wound gutured, remainder repasted. Two day's later there was slight purulent discharge from the upper end of the wound ; second foreign body localized by $x$ rays and removed ; wound repasted.

August 10th. Remainder of wound sutured.

August 14th. Wound healed.

Case 49.-Pte. G. G.; admitted August 2nd, 1917. Bullet wound of forearm with compound fracture of radius. Primary suture. On August 7th the wound was doing well; there was no suppuration. On the 12 th it was healed, and the patient was evacuated to the base.

Case 57.-Cpl. W. L. ; admitted August 7th, 1917. Shell wound of forearm with compound fracture of radius; much loss of blood prior to admission. On Angust loth the wound was repasted and sutured; on the 16th it was clean and healing, and the patient was evacuated to the base.

Case 61.-Pte. A. S.; admitted August 9th, 1917. Shell wounds of both forearms, with compound fractures of right ulna and left radius. On August 12th both wounds were sutured. On the 16th they were clean and healing, and the patient was evacuated to the base.

Case 62.-Pte. J. R.; admitted August 9th, 1917. Accidental injury: compound fracture of tibia and fibula. On August 15th the wound was perfectly clean.

Class II.-Successful.

Case 2.-Pte. E. H.; admitted July 8th, 1917. Shrapnel wounds (penetrating) of knee, with fracture, and of thigh (missile embedded in femur). Patient too collapsed for opera tion on admission. On recovery, amputation of feft thigh (lower third); the right thigh treated by other method as control. On July 17th the wounds were repasted and amputation flaps sutured.

July 25th. Healing; no discharge. Evacuated to base. Report from base: Sutures removed; wound healed; slight suppuration from right thigh (control).

Case 3.-Pte. T. G. ; admitted July 10th, 1917. Bullet wound of leg with division of peroneal vessels. On July 13th secondary suture was performed; on the 18th the sutures were removed when there was partial gaping of a clean wound. On the 25th he was evacuated to the base with the wound clean.

Case 4.-Pte. H. L.; admitted July 10th, 1917. Shell wound of leg, with compound comminuted fracture of tibia and fibula and division of popliteal artery. Very collapsed on admission. Supracondylar amputation ; flaps loosely sutured.

July 12th. Slight reactionary haemorrhage; clot turned out and wound repasted. This was followed by slight purulent discharge for a few days.

July 20th. Edges of flap freshened and sutured with drainage. Drain removed two days later.

July 30 th. Evacuated to base; wound healing.

Case 5.-Pte. H. G.; admitted July 11th, 1917, with extensive gutter wound of thigh through muscles. On July 18th the 
skin was approximated by sutures, but the wound could not be completely closed owing to loss of tissue.

July 22nd. Evacuated to base. Wound clean.

Case 6.-Cpl. W. O.; admitted July 11th, 1917, with shell wound of shoulder, superficial. On the 13th secondary suture was performed. He returned to duty on July 3lst with a sound scar.

Case 11.-Pte. W. L.; admitted July 12th, 1917, with shell wound of back. Primary suture. Evacuated to base on July 22nd with the wound healed.

Case 12.-Pte. D. S.; admitted July 12th, 1917, with bullet wound of shoulder. On July 14th secondary suture was performed; on the 20 th the wound was clean and healing, and the patient was evacuated to the base.

Case 13.-Pte. W. J.; admitted July 12th, 1917, with the following shell wounds : (1) Chest, opening pleura ; (2) scapular region; (3) arm ; (4) penetrating head (pasted and cleaned only) July 20th. All wounds clean; no suppuration; no head symptoms. Evacuated to base.

Case 14.-Spr. G. B.; admitted July 13th, 1917. Shell wound of back of thigh twenty-four hours before admission. Femur exposed but not fractured. Wound repasted and sutured on July 18th. Patient evacuated to base on July 25th, wound healing.

Case 18.-Pte. A. W.; admitted July 15th, 1917, with shell wounds of both legs. On July 21st secondary suture was per formed, and on the 27 th the wounds were healing, and the patient was evacuated to the base.

Case 19.-Pte. H. R.; admitted July 15th, 1917, with shell wound of back. Secondary suture was performed on July 19th, and three days later the patient was evacuated to the base with the wound healing.

Case 20.-Lce.-Cpl. M. W.; admitted July 15th, 1917, with shell wound of leg. Primary suture. On July 22 nd the wound was healing, and patient was evacuated to the base.

Case 21.-Pte. O. M. ; admitted July 15th, 1917, with shell wound of buttock. Secondary suture on July 21st. On the 25th wound healing, and patient evacuated to the base.

Case 22.-Pte. W. L.; admitted July 18th, 1917, with shell wounds of arm and both legs and thigh. On July 22nd the wounds were clean and closed with strapping. Patient evacuated to base on August lst with the wounds healing.

Case 24.-Pte. C. G.; admitted July 18th, 1917, with shell wound of buttock. Two days later the wound was clean and patient was evacuated to the base.

Case 25.-Pte. J. H. ; admitted July 19th, 1917. Shell wound of thigh and hand with compound fracture of metacarpals. Patient evacuated to base on August lst with the wounds clean.

Case 26.--Pte. J. C. ; admitted July 20th, 1917, with shell wound of leg. On the 25 th the wounds were clean, and patient was evacuated to the base.

Case 27.-Pte. P. S.; admitted July 21st, 1917, with shell wound of buttock. Foreign body not removed. Patient evacuated to base on August 30th with the wound clean.

Case 36.-Lieut. E. G. E. ; admitted July 28th, 1917. Bomb wounds of both legs, with gas gangrene of right. Very collapsed on admission. Direct blood transfusion. Flush ampu tation through right knee-joint.

August 1st. Amputation redressed under gas; wounds clean. Evacuated to base three days later with the wounds still clean.

Case 37.-Pte. J. V.; admitted July 28th, 1917, with shell wound of left arm. On the following day blood clot turned out and wound repasted. Secondary suture performed on August 3rd. Patient evacuated to base on August 12th with the wound healing.

Case 38.-Pte. H. B. ; admitted July 29th, 1917. Shell wound of face with compound fracture of maxilla. Evacuated to base on August lst; wounds clean.

Case 41.-Pte. C. B.; admitted July 30th, 1917, with shell wound of leg, exposing bone. Secondary suture on August lst. Wound healed on August 12th, and patient evacuated to base.

Case 42.-Pte. G. D.; admitted July 30th, 1917, with shell wound of back. Primary suture. Stitches removed on August 7 th ; wound healed. Evacuated to base on August 12th ; satisfactory.

Case 44.-Pte. G. P.; admitted July 31st, 1917. Old shell wound of thigh, with persistent sinus. Foreign body removed from side of femur by counter-incision; sinus excised. Secondary suture on August 2nd. On August 12th the patient Secondary suture on August 2nd. On August 12th
was evacuated to the base with the wound healed.

Case 45--Pte. F. D.; admitted July 31st, 1917, with shell wounds of arm and thigh. Primary suture of thigh. Secondary suture of arm on August 2nd. Stitches removed from thigh on August 7th; wound dry and healed. Evacuated to base on August 12 th with both wounds healed.

Case 46.-Pte. J. F.; admitted July 31st, 1917. Shell wound in occipital region, with damage to bones. Primary suture. Stitches removed on August 12th; wound healed; patient ovacuated to base.
Case 50.-Pte. P. J.; admitted August 2nd, 1917. Shell wound of hand, with compound fracture of metacarpal. Available tissue sutured two days later. Patient evacuated to base on August 12th with the wound clean.

Case 51.-Pte. P. C.; almitted August 3rd, 1917, with shell wound of buttock. Primary suture. The wound was healed on August 15th, and patient was evacuated to the base.

Case 52.-Lce.-Cpl. R. O.; admitted August 5th, 1917, with trench mortar wounds of forehead and arm. Primary suture of both wounds.

August 11th. Sutares removed from head; wound healed. Arm healing. Evacuated to base.

Case 53.-Pte. J. H. ; admitted August 5th, 1917, with shell wound of head penetrating brain. Primary suture.

August 16th. Stitches removed; wound healed. Still in hospital, awaiting train.

Case 54.-Lce.-Cpl. S. A.; admitted Angust 6th, 1917, witli shell wounds of back and leg. Secondary suture four days later. On August 16th wounds clean ; awaiting train.

Case 55.-Lce.-Cpl. E. D.; admitted August 6th, 1917, with shell wound of scalp. Primary suture. Patient evacuated to base on August 12th with the wound healed.

Case 56.-Dvr. H. T. ; admitted August 7th, 1917, with shell wound of shoulder. Long track only partially excised owing to exposure of brachial plexus. Patient evacuated to base on August 12th with the wound clean.

Case 58.-Pte. J. G.; admitted August 8th, 1917, with shell wounds of shoulder and leg. Primary suture of leg. Secondary suture of shoulder three days later: On August 16th botli wounds were clean and healing; patient awaiting train.

Case 59.-Dvr. M. M. ; admitted Ausust 8th, 1917, with shell wound of head. Primary suture. Patient evacuated to base on August 12th with the wound healing.

Case 60.-Pte. W. T.; admitted August 9th, 1917, with shell wound of chest wall. Primary suture.

August 16th. Healing. A waiting train.

Case 63.-Pte. H. S. ; admitted August 10th, 1917, with shell wound of thigh. Sutures inserted but not tied. Sutures tied on August 11th. Or the 16th the wound was healing and patient awaiting train.

Case 64.-Lce.-Cpl. L. F. ; admitted August 10th, 1917, with shell wound of thigh. Primary suture. Un August 16th the wound was dry and healing, and patient awaiting train.

Class III.-Relatively Unsuccessful.

Case 8.-Sgt. B. ; admitted July 11th, 1917. Shell wound of back, with compound comminuted fracture of both scapulae. Very extensive wounds, excised as far as possible. Patient extremely ill for several days. Both wounds suppurated, but settled down under fomentations without any further operative interference.

July 30th. Evacuated to base. Wounds granulating.

Case 9.-Pte. J. K. ; admitted July 12th, 1917, with the follow. ing shell wounds: (1) Heel-one foreign body removed from os calcis and one left embedded in bone; (2) leg-foreign boly not found in calf muscles; (3) forearm-two compound fractures of ulna; wounds partially excised; (4) hand-one finger blown ulna; wounds partially excised; (4) hand-one finger blown away; stump trimmed. The patient's conchition was so serious with spreading sepsis in forearm.

July 17th. Further incisions in arm; leg requirea no July 30th. Condition settled; still some mild suppuration. Evacuated to base.

Case 10.-Pte. J. D.; admitted July 12th, 1917, with shell wounds of both buttocks, one involving the thigh. Foreign body retained in thigh. Wounds already stinking. Death took place two days later. The autopsy showed extensive gas gangrene.

Case 17.-Pte. J. W.; admitted July 14th, 1917, with extensivo shell wound of thigh involving femur. Patient exsanguined. Death took place the same day, within twelve hours of operation. Patient never rallied and died from shock and haemorrhage. The autopsy showed no sepsis.

Case 23.-Lieut. H. L.; admitted July 17th, 1917. Shell wound of leg, with compound fracture of tibia and fibula; both sets of tibial vessels subsequently found to be divided. Patient shocked and exsanguined. Hurried operation.

July 18th. Gas gangrene in leg. Guillotine amputation at knee-joint; stump pasted.

July 25th. No further sepsis. Evacuated to base.

Case 28.-Pte. C. A. ; admitted July 22nd, 1917, with bullet wound of the chest wall and arm, dividing ulnar nerve.

July 26th. Arm remained quite clean; chest suppurated. Temperature unaffected. Cleaned up in five days with CarrelDakin treatment.

August lst. Wounds clean. Evacuated to base.

Case 29.-Pte. H. S., admitted July 23rd, 1917. Shell wound of chest, with compound fracture of scapula. Primary suture. Clean for four days, then slight purulent discharge. Stitches removed; Carrel-Dakin treatment.

August 2nd. Wound clean. base. ugust 12th. Approximated with strapping. Evacuated to 
Case 30.-Pte. F. M. ; admitted July 24th, 1917. Large shell wound of leg, with compound fracture of tibia and division of posterior tibial vessels and nerve; extensive laceration of muscles. Amputation. Wound clean for four days, then profuse purulent discharge. Carrel-Dakin treatment.

August 7tb. Clean but huge open wound. Patient evacuated to base on August 12th.

Case 33.-Pte. S. W. ; admitted July 25th, 1917. Shell wound of thigh, with compound fracture of femur and whole of back of thigh blown away. Extreme shock, contraindicating general ansesthetic other than nitrous oxide. No excision of wound only pasting. The following day the wound was stinking, and the patient's condition prevented operative treatment.

July 29th. Death from shock and sepsis.

Case 43.-Pte. W. G.; admitted July 30th, 1917, with the following shell wounds: (1) Back, with fractured spine and paralysis of bladder; (2) head, penetrating brain. Primary suture of head.

August 7th. Wounds clean.

August 12th. Head wound healed; back wound clean. Pyuria.

August 16th. Death. Autopsy showed sepsis of whole urinary track. Wounds clean.

\section{THE USE OF LIQUID PARAFFIN IN THE TREATMENT OF WAR WOUNDS. BY}

Temporary Colonel H. M. W. GRAY, C.B., A.M.S., consultant bURgen.

Durivg the past year much clinical observation has been carried out in several Casualty Clearing Stations in this Army on the effect of liquid paraffin upon wounds at different stages.

The results of these observations enable one to state:

1. That liquid paraffin has, almost without exception, a very beneficial action on the walls of a wound and on the tissues adjacent to the wound. It prevents develop. ment of the symptoms of inflammation in these parts and will cause such symptoms to disappear rapidly if they are already present. The skin remains or becomes normal in appearance; redness and swelling do not develop or, if present, disappear. Pain is assuaged. Performance of function becomes easier, although, of course, this is frequently restrained by therapeutic agencies and is therefore not evident. Changes of dressings are comparatively painless.

One does not care at this stage to discuss possible explanations of these effects of the paraffin, but it may be pointed out that the "electrical " conditions of wounds have usually hitherto been entirely disregarded, only. chemical and mechanical conditions having been considered. Electrical conditions of wounds, as revealed by the galvanometer, are found to vary, in a very consistent way, with the age of the wound and the amoun of inflammation present.

2. Liquid paraffin is not antiseptic in the ordinary sense of the term. If a wound be " packed" with gauze wrung loosely out of the, paraffin, it is found that in two to four days the discharge in the wound swarms - with organisms, although the surrounding parts are free from inflamma. tion. The "wound" with the fluid part of its contents may be likened to a test tube containing nutritive medium in which organisms are thriving. In either case the addition of antiseptic will lessen or entirely inhibit the growth. If an antiseptic is dissolved or suspended in the liquid paraffin with which the gauze pack is saturated, it is found that the wound remains or becomes sterile. - Various antiseptics or mixtures of antiseptics have been used, with good effects in all cases, although these effects vary, possibly owing to selective effect of the particular antiseptic against the particular organism or to tolerance acquired by the organisms for the antiseptic omployed against them. Those chiefly used up to the present are flavine, brilliant green, boric acid, and iodoform. It is rather difficult to justify preference for any one. A com. bination seems desirable. Non-poisonous antiseptics with more or less prolonged action when in contact with body fluids are likely to be most successful. It seems that, although the effects of micro-organisms on the tissues adjacent to a wound are inhibited, absorption of chemical antiseptics from the wound may occur and cause symptoms of poisoning.

One would desire to draw attention to papers sent for publication by Captain W. H. Hey and others (p. 506), which deal with clinical results obtained from the use of pastes made with liquid paraffin as a base. Captain Hey found that pastes made with other substances-for example, gums-are inferior in curative action to those made with paraffin. During his investigations Captain Hey did not regard the bases with which his various pastes were made up as being anything else than " neutral."

It has been stated that the paraffin in bipp paste delays healing. Delay in healing of recent wounds, as has been observed in "bipped" cases, has not occurred in paraffin pastes made up with antiseptics soluble in serum. It seems more likely that the excess of bismuth subnitrate and iodoform in bipp is responsible by preventing adhesion of apposed surfaces.

The only type of inflammation, in cases which are not already too far advanced to recover, which in $\mathrm{my} e x$ perience has not yielded to the free application of liquid paraffin is that exemplified in a wounded limb which becomes much swollen, brawny, grossly nodular, and brownish-red, the wound being covered with granulations which look like dirty pale pink frog's spawn.

In all cases it is essential for success to have thorough preliminary opening up and mechanical cleansing of the wound. A piece of half-dead muscle left behind, infected with gas bacilli, is not likely to become disinfected by antiseptics, especially when the patient's resisting powers are at a low ebb; the paraffin is likewise at a disadvantage because the affected tissues cannot react in the ordinary way. Gauze used for packing a wound smeared with a paraffin paste should be wrung out of liquid paraffin or iodoform-paraffin (1 per cent.), and the skin around the wound covered with similarly impregnated ganze. Captain Hey, Captain Short, Captain J. Anderson, and others have shown that wounds may be sutured with success at a very early stage after treatment by such methods.

\section{REINFECTION IN SYPHILIS: WITH OBSERVATIONS ON 28 CASES.}

BY

\section{CHARLES F. WHITE, MaJor R.A.M.C.}

Probably, though it would be a question difficult to settle, there is no such thing as a natural immunity from syphilis. However this may be, it was held until recently that immunity from syphilis might be acquired. The older theory, now abandoned, was that one infection of syphilis produced immunity from further infection; in other words, that syphilis could be acquired once only in a lifetime. Careful investigation appears to establish-

1. That reinfection can take place.

2. That such reinfection cannot take place until the original infection has been completely cured.

With the older treatment of syphilis a complete cure was difficult, or very nare, and consequently cases of rein. fection were so exceptional that a doubt of their existence was not unreasonable.

The collection and examination of instances of reinfection are therefore of great importance. First, as showing beyond doubt that reinfection is a fact, and, secondly, as amounting to almost complete proof of the cure of the first attack.

Since the introduction of the combined salvarsan and mercury treatment for syphilis the number of cases of genuine reinfections reported has increased-a sure sign of the curative efficacy of this treatment and a proof that one attack of syphilis does not produce immunity. Perhaps we should not be far wrong in saying that the only persons immune from syphilis are the syphilitics.

Formerly proof of reinfection was based on clinical evidence alone and was open to certain fallacies, namely:

(a) In the Case of the First Attack.-Absence of positive proof of syphilis in those cases in which treatment was started in the primary stage and in which secondary symptoms did not develop. As no microscopic examination was made, or in the failure to find spirochaetes in serum from the sore, it was always possible that the diagnosis was mistaken. In other words, there was. no positive. proof that the chancre was syphilitic and not a soft sore.

(b) In the Case of the Second Attack.-(1) A soft chancre, (2) a recurrent chancre, (3) a chancriform gumma, or 